\section{Fatores individuais e ambientais associados com o uso de bicicleta por adultos: uma revisão sistemática}

\author{
Individual and environmental factors \\ associated with bicycling by adults: a \\ systematic review
}

\author{
Marilson Kienteka ${ }^{1,3}$ \\ Rogério César Fermino ${ }^{1,2}$ \\ Rodrigo Siqueira Reis 1,2,3 $^{1,2}$
}

\section{RESUMO}

O objetivo desta revisão foi identificar os fatores individuais e ambientais associados com o uso de bicicleta por adultos. Foram selecionados estudos originais indexados nas bases Lilacs, PubMed, Science Direct e Medline e a busca foi realizada com a combinação dos descritores em inglês estabelecidos pelo Medical Subject Headings ( $\mathrm{MeSH})$ e em português pelo Descritores em Ciências da Saúde (DeCS) (ex: "bicycling", "motor activity", "transportation", "leisure activities", "commuting”). A revisão foi finalizada em agosto de 2011. Vinte e nove estudos publicados em inglês atenderam aos critérios de inclusão e foram identificados 57 fatores potencialmente associados com o uso de bicicleta. Entre os fatores individuais, sexo masculino e prática de atividade física em níveis recomendados ( $\geq 150 \mathrm{~min} / \mathrm{sem})$ apresentaram associação positiva conclusiva com o uso da bicicleta. Ainda, o trabalho de oito horas e a posse de carro apresentaram associação negativa conclusiva com o desfecho. Entre os fatores ambientais, acesso a ciclovias/ciclofaixas, densidade de ruas, moradia urbana e disponibilidade de bicicleta no ambiente domiciliar apresentaram associação positiva conclusiva com o uso de bicicleta. No entanto, distância para os destinos superiores a quatro km e inclinação das ruas apresentaram associação negativa conclusiva com o desfecho. Estes resultados podem auxiliar na elaboração de políticas públicas com o planejamento de intervenções e benfeitorias que favoreçam o uso de bicicleta pela população.

\section{PALAVRAS-CHAVE}

Ciclismo; Atividade Motora; Exercício; Recreação.

\begin{abstract}
The aim of this review was to identify individual and environmental factors associated with bicycle use in adults. We selected original studies indexed in Lilacs, PubMed, Science Direct, Medline and the search was conducted with combination of descriptors in English established by the Medical Subject Headings (MeSH) and Portuguese by "Descritores em Ciências da Saúde (DeCS)" (e.g. "bicycling", "motor activity", "transportation", "leisure activities", "commuting"). Twenty-nine studies, published in English, met the inclusion criteria that were identified 57 factors potentially associated with the use of bicycle. Among the individual factors, male sex and physical activity practices in recommended levels ( $\geq 150 \mathrm{~min} /$ week) showed conclusive positive association with bicycle use. The work of eight hours and car ownership had a negative association conclusive with the outcome. Among the environmental factors, access to bike paths/lanes, streets density, availability of bicycle in home and urban housing was positively associated conclusive with bicycle use. However, distance to destinations more than four $\mathrm{km}$ and streets inclination had a negative association conclusive with the outcome. These results may help in the development of public policies with planning interventions and improvements that encourage bicycle use by the population.
\end{abstract}

Rev Bras Ativ Fis Saúde p. 12-24 DOI:

http://dx.doi.org/10.12820/rbafs.v.19n1p12

1 Grupo de Pesquisa em Atividade Física e Qualidade de Vida, GPAQ/PUCPR, Curitiba, PR, Brasil.

2 Curso de Educacão Física, Escola de Saúde e Biociências. Pontifícia Universidade Católica do Paraná - PUCPR

3Programa de Pós Graduação em Educação Física. Universidade Federal do Paraná UFPR. Curitiba-PR, Brasil.

\title{
KEYWORDS
}

Bicycling; Motor Activity; Exercise; Recreation. 


\section{INTRODUÇÃO}

Evidências apontam o elevado potencial que o uso da bicicleta pode apresentar para o aumento dos níveis de atividade física e redução de morbidades na população ${ }^{1,2}$. Além destes benefícios, o uso de bicicleta pode ter um impacto positivo no ambiente urbano e na economia, uma vez que a redução do número de veículos diminui os congestionamentos, a emissão de $\mathrm{CO}_{2}$ e o consumo de combustíveis ${ }^{3}$. O uso de bicicleta em larga escala também tem elevado potencial para a saúde pública uma vez que seu custo reduzido a torna acessível para a população ${ }^{2}$.

O uso da bicicleta no deslocamento e no lazer parece variar entre os paises ${ }^{4-6}$. No Brasil, aproximadamente um em cada dez adultos reportam este comportamento ${ }^{5,6}$. Esta proporção é similar àquelas observadas no Canadá (8$10 \%)^{2,7,8}$ e Austrália $(6-14 \%)^{9,10}$, mas inferior ao verificado em países europeus como Holanda (26-29\%) $)^{1112}$ e Áustria $(23-41 \%)^{13,14}$. Estas diferenças podem ser parcialmente explicadas pelos diversos fatores associados com o uso de bicicleta, além de questões culturais.

De fato, evidências apontam que fatores individuais (sexo, idade, renda, prática de atividade física, etc.) psicossociais (segurança, tempo de deslocamento, custo, etc.) e ambientais (características do bairro, uso do solo, presença de estruturas para bicicleta, etc.) estão associados com o uso de bicicleta $^{15,16}$. Em geral, este comportamento é mais comum entre os homens ${ }^{4,9,10}$, indivíduos mais jovens ${ }^{2,7,17}$, com menor renda ${ }^{2,8,18}$ e com percepção positiva de saúde $^{19}$. Além destas características, aspectos relacionados ao ambiente como a proximidade e acesso a ciclovias ${ }^{9}$, proximidade de destinos ${ }^{20}$, terreno plano ${ }^{8}$ e o acesso a bicicletas parecem favorecer o uso de bicicletas.

Apesar destas evidências, não foram identificadas revisões da literatura que tenham sintetizado as informações de estudos quantitativos sobre a consistência dos fatores individuais e ambientais associados com o uso de bicicleta por adultos. Estas informações podem auxiliar no direcionamento de intervenções de profissionais da área de saúde pública, planejamento urbano e engenharia, entre outros, com o intuito de facilitar este comportamento ${ }^{21}$. O objetivo desta revisão foi sintetizar as evidências sobre os fatores individuais e ambientais associados com o uso da bicicleta no deslocamento e no lazer por adultos.

\section{MÉTODOS}

A revisão sistemática seguiu os procedimentos descritos na literatura ${ }^{22}$ e foi realizada nas principais bases de dados da área de saúde: Lilacs, PubMed, Science Direct e Medline. Foram incluídos apenas estudos que atenderam os seguintes critérios de inclusão: a) estudos observacionais empíricos com delineamento transversal ou longitudinal; b) com análises quantitativas; c) contendo desfecho sobre uso de bicicleta; d) realizados com adultos ( $\geq 18$ anos); e e) publicados em língua portuguesa ou inglesa. Foram excluídos os estudos de a) natureza qualitativa; b) revisões de literatura; c) realizados exclusivamente com crianças e/ou adolescentes; d) atletas ou indivíduo com morbidades; e) cujos desfechos caracterizassem acidentes, lesões ou mortes relacionadas ao uso de bicicleta; e ainda f) artigos de opinião ou carta para o editor; ou livros, capítulos, dissertações e teses. 
Os limites para a busca incluíram pesquisas conduzidas em humanos e publicadas a partir de 1980. Esta delimitação de data é sugerida por estudos que apontam o número limitado de evidências anteriores a década de $1990^{23}$ e também por este período ser indicado como o ponto de partida dos estudos em ciências comportamentais $^{24}$. A busca foi realizada entre os meses de julho e agosto de 2011 com a combinação dos descritores padronizados pelo Medical Subject Headings (MeSH) nas bases de dados PubMed, Science Direct e Medline ("bicycling", "motor activity", "exercise", "recreation", "transportation", "leisure activities", "pendular migration" e "commuting"). Foram utilizados os seus correspondentes em língua portuguesa, padronizados pelo Descritores em Ciências da Saúde (DeCS) para a busca na base de dados Lilacs ("ciclismo", "atividade motora", "atividade física", "exercício", "recreação", "transporte”, "lazer" e "pendular”). As buscas foram realizadas com a utilização dos operadores booleanos "AND" e "OR".

A figura 1 apresenta o fluxograma da busca, seleção e os respectivos motivos de exclusão dos estudos. A seleção e leitura dos artigos, assim como a extração de informações, foi realizada por dois avaliadores familiarizados coma metodologia de revisão sistemática ${ }^{22}$. Foram identificados e registrados os aspectos gerais da publicação (ano, cidade e país), características metodológicas (participantes, métodos empregados, delineamento do estudo) e principais resultados dos estudos (associações testadas). A triagem das informações foi realizada de maneira independente entre os revisores, e posteriormente comparada em reunião de consenso. Os itens que apresentaram concordância entre os revisores foram considerados adequados e incluídos na descrição dos resultados. Os itens discordantes foram revisados pelo pesquisador principal, discutido em uma nova reunião de consenso para finalmente serem incluídos nos resultados.

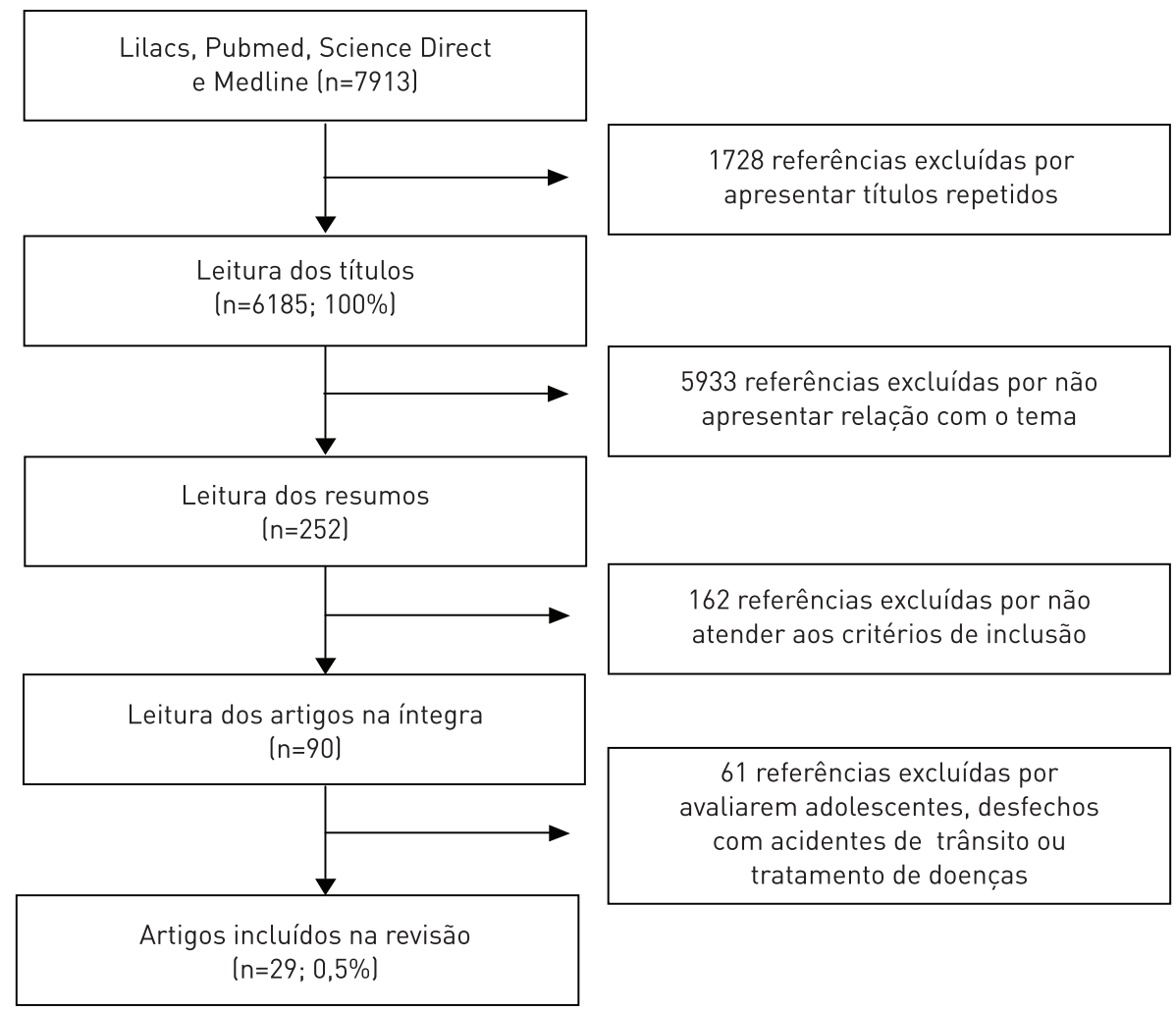

FIGURA 1 - Fluxograma de busca, seleção e exclusão dos artigos para a revisão. 
Os estudos foram comparados para determinar a consistência entre os resultados (tabela 1). Inicialmente foi estabelecida a razão entre o número de estudos com resultados demonstrando a mesma direção de associação e o total de estudos identificados. Em seguida os resultados foram classificados segundo a consistência de achados adotando os procedimentos utilizados em outras revisões na área de atividade física ${ }^{25-27}$. Considerou-se associação conclusiva quando uma variável foi analisada em pelo menos três estudos e quando a concordância de resultados foi entre $60-100 \%$. Associação inconclusiva foi atribuída quando a variável foi reportada em pelo menos três estudos e com concordância entre 34-59\%. Por fim, a associação inconsistente foi considerada quando a variável apresentou menos de três estudos apoiando o mesmo sentido de associação (tabela 1).

TABELA 1 - Classificação utilizada para a síntese das evidências.

\begin{tabular}{lcc}
\hline \% de estudos que suportam a associação & Código & Associação \\
\hline $0-33 \%^{\mathrm{a}}$ & $? ?$ & Inconsistente \\
\hline $34-59 \%^{\mathrm{b}}$ & $?$ & Inconclusiva \\
\hline \multirow{2}{*}{$\geqslant 60 \%^{\mathrm{b}}$} & + & Conclusiva \\
\cline { 2 - 3 } & - & Conclusiva \\
\hline
\end{tabular}

${ }^{\text {a }}$ ou $<3$ estudos: ${ }^{\text {b }}$ e $\geqslant 3$ estudos.

\section{RESULTADOS}

\section{Características gerais}

Vinte e nove estudos atenderam aos critérios de inclusão e foram publicados entre os anos de 2003 e 2011. A maior parte dos estudos foi realizada na América do Norte $(\mathrm{n}=12 ; 41 \%)$, seguida da Europa ( $\mathrm{n}=9 ; 31 \%)$, Oceania $(\mathrm{n}=6 ; 21 \%)$ e América do Sul $(\mathrm{n}=2 ; 7 \%)$. A maioria dos estudos apresentou delineamento transversal $(\mathrm{n}=28 ; 96 \%)$. Ao todo 19 estudos $(66 \%)$ incluíram amostragens probabilísticas, dos quais, sete estudos utilizaram amostras representativas $2,4,7,19,31,32,36$. Demais estudos empregaram amostras por conveniência $8,10,21,30,34,35,38,40,42$ ou não informaram o critério de seleção $0^{37}$.

Todos os estudos contemplaram ambos os sexos e $83 \%$ apresentaram indivíduos adultos em suas amostras. No entanto, alguns estudos incluíram adolescentes $(17 \%)$ e foram mantidos na revisão, por representarem a minoria do total da amostra e também pela necessidade de manter um maior número de evidências na revisão.

A frequência de uso da bicicleta foi maior na Europa (10,1-63,5\%), seguida da Oceania (5,8-42,7\%), América do Norte (0,6-21,3\%) e América do Sul $(11,2-15 \%)$.

$\mathrm{O}$ uso da bicicleta foi mensurado de diferentes maneiras. Em 18 estudos (62\%) foram empregadas questões específicas para cada desfecho, porém seis estudos $(20,5 \%)$ utilizaram questões padronizadas de instrumentos como o International Physical Activity Questionnaire (IPAQ) e o Flemish Physical Activity.

Todos os estudos apresentaram informações sobre o uso da bicicleta no deslocamento, no entanto, três destes reportaram resultados de forma geral (deslocamento + lazer) ${ }^{28,32,39}$ e outros três contemplaram o uso da bicicleta no lazer separadamente $e^{4,29,33}$. 
TABELA 2 - Métodos e características dos estudos incluídos na revisão segundo o país de origem e ano de publicação.

\begin{tabular}{|c|c|c|c|c|c|c|c|c|c|}
\hline Autor & País & Delineamento & Freq. & Sexo & $\mathrm{n}$ & Métodos & $\begin{array}{l}\text { Faixa } \\
\text { etária }\end{array}$ & Fatores individuais & Fatores ambientais \\
\hline $\begin{array}{l}\text { Winters et } \\
\text { al. }(2007)^{7}\end{array}$ & Canadá & Transversal & $7,9 \%$ & $M, F$ & 59.899 & $\begin{array}{l}\text { Inquérito } \\
\text { telefônico }\end{array}$ & $>12$ & $\begin{array}{l}\text { Sexo, idade, renda, } \\
\text { escolaridade }\end{array}$ & $\begin{array}{l}\text { Dias por ano de chuva e } \\
\text { temperaturas }\end{array}$ \\
\hline $\begin{array}{l}\text { Butler et al. } \\
(2007)^{2}\end{array}$ & Canadá & Transversal & $\begin{array}{l}\text { Masc. } \\
10,3 \% \\
\text { Fem. } \\
5,7 \%\end{array}$ & $M, F$ & 77.953 & $\begin{array}{l}\text { Inquérito } \\
\text { telefônico }\end{array}$ & $>15$ & $\begin{array}{l}\text { Sexo, idade, renda, } \\
\text { escolaridade, estado } \\
\text { civil, tabagismo, } \\
\text { nível de atividade } \\
\text { física }\end{array}$ & $\begin{array}{l}\text { Região, área de } \\
\text { residência }\end{array}$ \\
\hline $\begin{array}{l}\text { Winters et } \\
\text { al. }(2010)^{8}\end{array}$ & Canadá & Transversal & $7,7 \%$ & $M, F$ & 1902 & $\begin{array}{l}\text { Inquérito } \\
\text { telefônico }\end{array}$ & $>19$ & $\begin{array}{l}\text { Sexo, idade, renda, } \\
\text { escolaridade, status } \\
\text { de trabalho }\end{array}$ & $\begin{array}{l}\text { Inclinação das ruas, } \\
\text { densidade de interseções, } \\
\text { estrutura para uso de } \\
\text { bicicleta, densidade } \\
\text { populacional }\end{array}$ \\
\hline $\begin{array}{l}\text { Cervero } \\
\text { e Duncan } \\
(2003)^{20}\end{array}$ & $\begin{array}{l}\text { Estados } \\
\text { Unidos }\end{array}$ & Transversal & $2,3 \%$ & $M, F$ & 15.066 & $\begin{array}{l}\text { Inquérito } \\
\text { domiciliar }\end{array}$ & $\begin{array}{c}\text { Não } \\
\text { informado }\end{array}$ & Sexo, renda & $\begin{array}{l}\text { Distância das viagens, } \\
\text { inclinação das ruas, } \\
\text { posse de carro, posse } \\
\text { de bicicleta no domicílio, } \\
\text { características do } \\
\text { ambiente construído }\end{array}$ \\
\hline $\begin{array}{l}\text { Sharpe et } \\
\text { al. }(2004)^{28}\end{array}$ & $\begin{array}{l}\text { Estados } \\
\text { Unidos }\end{array}$ & Transversal & -- & $M, F$ & 1936 & $\begin{array}{l}\text { Inquérito } \\
\text { telefônico }\end{array}$ & $>18$ & $\begin{array}{l}\text { Sexo, idade, renda, } \\
\text { escolaridade, raça }\end{array}$ & $\begin{array}{l}\text { Rotas mapeadas para } \\
\text { andar de bicicleta }\end{array}$ \\
\hline $\begin{array}{l}\text { Plaut } \\
(2005)^{18}\end{array}$ & $\begin{array}{l}\text { Estados } \\
\text { Unidos }\end{array}$ & Transversal & $0,6 \%$ & $M, F$ & 38.314 & $\begin{array}{l}\text { Inquérito } \\
\text { online }\end{array}$ & $\begin{array}{l}28 \% \leqslant 25 \\
72 \% \geqslant 26\end{array}$ & $\begin{array}{l}\text { Sexo, idade, renda, } \\
\text { escolaridade }\end{array}$ & $\begin{array}{l}\text { Tipo de habitação, posse } \\
\text { de carro }\end{array}$ \\
\hline $\begin{array}{l}\text { Moudon et } \\
\text { al. }(2005)^{29}\end{array}$ & $\begin{array}{l}\text { Estados } \\
\text { Unidos }\end{array}$ & Transversal & $21,3 \%$ & $M, F$ & 608 & $\begin{array}{l}\text { Inquérito } \\
\text { telefônico }\end{array}$ & $>18$ & $\begin{array}{l}\text { Sexo, idade, renda, } \\
\text { estado civil, raça, } \\
\text { estado de saúde }\end{array}$ & $\begin{array}{l}\text { Quantidades de destinos } \\
\text { dentro de um buffer } \\
\text { de } 3 \mathrm{~km} \text {, densidade } \\
\text { residencial, tamanho da } \\
\text { quadra/rua, comprimento } \\
\text { da calçada, volume e } \\
\text { velocidade de tráfego, } \\
\text { topografia, posse de } \\
\text { carro e disponibilidade de } \\
\text { bicicleta no domicílio }\end{array}$ \\
\hline $\begin{array}{l}\text { Heesch e } \\
\text { Han }(2007)^{30}\end{array}$ & $\begin{array}{l}\text { Estados } \\
\text { Unidos }\end{array}$ & Transversal & -- & $M, F$ & 460 & $\begin{array}{l}\text { Inquérito } \\
\text { telefônico }\end{array}$ & $>18$ & $\begin{array}{l}\text { Sexo, idade, renda, } \\
\text { escolaridade, status } \\
\text { de emprego, estado } \\
\text { civil, filhos }<18 \\
\text { anos, IMC }\end{array}$ & $\begin{array}{l}\text { Área de residência, } \\
\text { suporte na criação de } \\
\text { ciclovias }\end{array}$ \\
\hline $\begin{array}{l}\text { Zahran et } \\
\text { al. }(2008)^{31}\end{array}$ & $\begin{array}{l}\text { Estados } \\
\text { Unidos }\end{array}$ & Transversal & $1,0 \%$ & $M, F$ & $\begin{array}{l} \pm 50 \\
\text { milhões } \\
\text { residências }\end{array}$ & $\begin{array}{l}\text { Inquérito } \\
\text { online }\end{array}$ & $>16$ & $\begin{array}{l}\text { Sexo, idade, } \\
\text { escolaridade, raça }\end{array}$ & $\begin{array}{l}\text { Ambiente construído, } \\
\text { densidade populacional, } \\
\text { poluição do ar }\end{array}$ \\
\hline $\begin{array}{l}\text { Rashad } \\
(2009)^{32}\end{array}$ & $\begin{array}{l}\text { Estados } \\
\text { Unidos }\end{array}$ & Transversal & $4,9 \%$ & $M, F$ & 146.730 & $\begin{array}{l}\text { Inquérito } \\
\text { telefônico }\end{array}$ & $>18$ & $\begin{array}{l}\text { Sexo, idade, renda, } \\
\text { escolaridade, estado } \\
\text { civil, e status de } \\
\text { emprego }\end{array}$ & $\begin{array}{l}\text { Expansão urbana, custo } \\
\text { do combustível, distância, } \\
\text { precipitação (chuva), } \\
\text { horas de sol }\end{array}$ \\
\hline $\begin{array}{l}\text { Xing et al, } \\
(2009)^{33}\end{array}$ & $\begin{array}{l}\text { Estados } \\
\text { Unidos }\end{array}$ & Transversal & $11,1 \%$ & $M, F$ & 965 & $\begin{array}{l}\text { Inquérito por } \\
\text { e-mail }\end{array}$ & $\begin{array}{c}\text { Não } \\
\text { informado }\end{array}$ & $\begin{array}{l}\text { Idade, renda, } \\
\text { escolaridade, } \\
\text { atitudes em direção } \\
\text { da bicicleta }\end{array}$ & $\begin{array}{l}\text { Distância, segurança, } \\
\text { cultura de ciclismo }\end{array}$ \\
\hline $\begin{array}{l}\text { Kaczinski et } \\
\text { al. }(2010)^{34}\end{array}$ & $\begin{array}{l}\text { Estados } \\
\text { Unidos }\end{array}$ & Transversal & -- & $M, F$ & 375 & $\begin{array}{l}\text { Inquérito } \\
\text { online }\end{array}$ & $>18$ & $\begin{array}{l}\text { Sexo, idade, } \\
\text { escolaridade, raça }\end{array}$ & $\begin{array}{l}\text { Presença de } \\
\text { estacionamento para } \\
\text { bicicleta, políticas de } \\
\text { armazenamento de } \\
\text { bicicleta em locais } \\
\text { variados e presença de } \\
\text { chuveiros e armários no } \\
\text { local de trabalho }\end{array}$ \\
\hline
\end{tabular}




\begin{tabular}{|c|c|c|c|c|c|c|c|c|c|}
\hline Autor & País & Delineamento & Freq. & Sexo & $n$ & Métodos & $\begin{array}{l}\text { Faixa } \\
\text { etária }\end{array}$ & Fatores individuais & Fatores ambientais \\
\hline $\begin{array}{l}\text { Parra et al. } \\
(2011)^{5}\end{array}$ & Brasil & Transversal & $8,0 \%$ & $M, F$ & 2.097 & $\begin{array}{l}\text { Inquérito } \\
\text { telefônico }\end{array}$ & $>18$ & $\begin{array}{l}\text { Sexo, idade, } \\
\text { escolaridade }\end{array}$ & $\begin{array}{l}\text { Walkability, percepção } \\
\text { de segurança (crimes e } \\
\text { trânsito), qualidade do } \\
\text { ambiente e percepção } \\
\text { de distância até destinos } \\
\text { comerciais no bairro }\end{array}$ \\
\hline $\begin{array}{l}\text { Cervero et } \\
\text { al. }(2009)^{4}\end{array}$ & Colômbia & Transversal & $15 \%$ & $M, F$ & 1.315 & $\begin{array}{l}\text { Inquérito } \\
\text { domiciliar }\end{array}$ & $>18$ & $\begin{array}{l}\text { Sexo, idade, renda, } \\
\text { saber andar de } \\
\text { bicicleta }\end{array}$ & $\begin{array}{l}\text { Conectividade e } \\
\text { densidade de ruas, } \\
\text { comprimento da ciclovia, } \\
\text { segurança no tráfego, } \\
\text { inclinação das ruas, } \\
\text { percepção de pessoas } \\
\text { que utilizam bicicleta e } \\
\text { posse de carro e bicicleta }\end{array}$ \\
\hline $\begin{array}{l}\text { Huy et al. } \\
(2008)^{19}\end{array}$ & Alemanha & Transversal & $44,6 \%$ & $M, F$ & 2002 & $\begin{array}{l}\text { Inquérito } \\
\text { telefônico }\end{array}$ & $50-70$ & $\begin{array}{l}\text { Sexo, idade, } \\
\text { escolaridade, } \\
\text { presença de riscos } \\
\text { médicos, percepção } \\
\text { de saúde }\end{array}$ & --- \\
\hline $\begin{array}{l}\text { Titze et } \\
\text { al.(2007) }\end{array}$ & Áustria & Transversal & $41,4 \%$ & $M, F$ & 634 & $\begin{array}{l}\text { Inquérito } \\
\text { universitário }\end{array}$ & $>18$ & $\begin{array}{l}\text { Sexo, idade, renda, } \\
\text { IMC, tabagismo, } \\
\text { nível de exercício, } \\
\text { satisfação } \\
\text { emocional, esforço } \\
\text { fisiológico }\end{array}$ & $\begin{array}{l}\text { Segurança no tráfego, } \\
\text { atratividade, segurança } \\
\text { para guardar a } \\
\text { bicicleta, mobilidade, } \\
\text { comportamento dos } \\
\text { colegas sobre uso da } \\
\text { bicicleta, disputa com um } \\
\text { carro }\end{array}$ \\
\hline $\begin{array}{l}\text { Titze et al. } \\
(2008)^{14}\end{array}$ & Áustria & Transversal & $22,5 \%$ & $M, F$ & 1.000 & $\begin{array}{l}\text { Inquérito } \\
\text { telefônico }\end{array}$ & $>15$ & $\begin{array}{l}\text { Sexo, idade, } \\
\text { escolaridade, IMC, } \\
\text { nível de atividade } \\
\text { física, criança em } \\
\text { casa <15 anos, } \\
\text { percepção de } \\
\text { benefícios: emoção } \\
\text { e rapidez, percepção } \\
\text { de barreiras: } \\
\text { desconforto } \\
\text { físico e modo } \\
\text { impraticável meio de } \\
\text { deslocamento }\end{array}$ & $\begin{array}{l}\text { Distância até os destinos, } \\
\text { conectividade de ciclovias, } \\
\text { apoio social para o uso de } \\
\text { bicicleta e posse de carro }\end{array}$ \\
\hline $\begin{array}{l}\text { De Geus et } \\
\text { al. }(2008)^{35}\end{array}$ & Bélgica & Transversal & $55 \%$ & $M, F$ & 343 & $\begin{array}{l}\text { Inquérito } \\
\text { online }\end{array}$ & $18-65$ & $\begin{array}{l}\text { Sexo, idade, } \\
\text { escolaridade } \\
\text { autoeficácia, } \\
\text { percepção de } \\
\text { benefícios e barreiras }\end{array}$ & $\begin{array}{l}\text { Destino, variáveis do } \\
\text { tráfego, estrutura para o } \\
\text { uso de bicicleta }\end{array}$ \\
\hline $\begin{array}{l}\text { Asztalos et } \\
\text { al. }(2009)^{36}\end{array}$ & Bélgica & Transversal & $17,6 \%$ & $M, F$ & 1919 & $\begin{array}{l}\text { Inquérito } \\
\text { online }\end{array}$ & $20-65$ & $\begin{array}{l}\text { Sexo, idade, status } \\
\text { ocupacional, } \\
\text { percepção de } \\
\text { estresse }\end{array}$ & $\begin{array}{l}\text { Status de emprego, uso } \\
\text { da bicicleta para ir ao } \\
\text { trabalho }\end{array}$ \\
\hline $\begin{array}{l}\text { Bruijn et al. } \\
(2009)^{37}\end{array}$ & Holanda & Transversal & $10,1 \%$ & $M, F$ & 317 & $\begin{array}{l}\text { Inquérito por } \\
\text { correio }\end{array}$ & $\begin{array}{c}42,35 \\
(D P \pm 0,87)\end{array}$ & $\begin{array}{l}\text { Sexo, idade, renda, } \\
\text { escolaridade, IMC e } \\
\text { status de emprego }\end{array}$ & --- \\
\hline $\begin{array}{l}\text { Heinen et } \\
\text { al. }(2010)^{11}\end{array}$ & Holanda & Transversal & $\begin{array}{l}26- \\
29 \%\end{array}$ & $M, F$ & 4299 & $\begin{array}{l}\text { Inquérito } \\
\text { online }\end{array}$ & $18-65$ & $\begin{array}{l}\text { Sexo, idade, renda, } \\
\text { estado civil, criança } \\
\text { em casa, percepção } \\
\text { de: relaxamento } \\
\text { mental e físico, } \\
\text { status social do } \\
\text { modal, conforto, } \\
\text { economia de tempo, } \\
\text { flexibilidade, baixo } \\
\text { custo, agradabilidade } \\
\text { como deslocamento, } \\
\text { estilo de vida }\end{array}$ & $\begin{array}{l}\text { Benefícios ambientais, } \\
\text { oferta de privacidade } \\
\text { pelo uso da bicicleta, } \\
\text { benefícios para a saúde, } \\
\text { segurança (tráfego e } \\
\text { pessoal) }\end{array}$ \\
\hline
\end{tabular}




\begin{tabular}{|c|c|c|c|c|c|c|c|c|c|}
\hline Autor & País & Delineamento & Freq. & Sexo & $n$ & Métodos & $\begin{array}{l}\text { Faixa } \\
\text { etária }\end{array}$ & Fatores individuais & Fatores ambientais \\
\hline $\begin{array}{l}\text { Engbers e } \\
\text { Hendriken } \\
(2010)^{12}\end{array}$ & Holanda & Transversal & -- & $M, F$ & 799 & $\begin{array}{l}\text { Inquérito } \\
\text { online }\end{array}$ & $18-65$ & $\begin{array}{l}\text { Sexo, idade, renda, } \\
\text { escolaridade, status } \\
\text { de emprego }\end{array}$ & Distância para o trabalho \\
\hline $\begin{array}{l}\text { Hendriksen } \\
\text { et al. } \\
(2010)^{38}\end{array}$ & Holanda & Transversal & $63,5 \%$ & $M, F$ & 1236 & $\begin{array}{l}\text { Inquérito } \\
\text { online }\end{array}$ & $32-53$ & $\begin{array}{l}\text { Sexo, idade, } \\
\text { escolaridade, nível } \\
\text { de atividade física, } \\
\text { percepção de saúde, } \\
\text { IMC, tabagismo }\end{array}$ & $\begin{array}{l}\text { Distância: } \leqslant 5,6-10 \text { e }>10 \\
\text { km. Velocidade: } \geqslant 16,17- \\
20 \text { e }>20 \text { km/h. Frequência } \\
\text { de uso de bicicleta (vezes } \\
\text { por semana) }\end{array}$ \\
\hline $\begin{array}{l}\text { Merom et } \\
\text { al. }(2003)^{39}\end{array}$ & Austrália & Coorte & -- & $M, F$ & 450 & $\begin{array}{l}\text { Inquérito } \\
\text { telefônico }\end{array}$ & $18-65$ & $\begin{array}{l}\text { Sexo, idade, status } \\
\text { civil, raça }\end{array}$ & $\begin{array}{l}\text { Distância de moradia até } \\
\text { uma trilha para uso de } \\
\text { bicicleta }\end{array}$ \\
\hline $\begin{array}{l}\text { Garrard et } \\
\text { al. }(2008)^{40}\end{array}$ & Austrália & Transversal & -- & $M, F$ & 6589 & $\begin{array}{l}\text { Observação } \\
\text { direta }\end{array}$ & -- & Sexo & $\begin{array}{l}\text { Presença de instalações } \\
\text { para o uso de bicicleta }\end{array}$ \\
\hline $\begin{array}{l}\text { Owen et al. } \\
(2010)^{10}\end{array}$ & Austrália & Transversal & $\begin{array}{l}14 \% \\
50 \%\end{array}$ & $M, F$ & $\begin{array}{c}1.940 \\
372\end{array}$ & $\begin{array}{l}\text { Inquérito por } \\
\text { correio }\end{array}$ & $\begin{array}{l}20-65 \\
18-65\end{array}$ & $\begin{array}{l}\text { Sexo, idade, } \\
\text { escolaridade, status } \\
\text { de emprego }\end{array}$ & Walkability \\
\hline $\begin{array}{l}\text { Rissel et al. } \\
(2010)^{41}\end{array}$ & Austrália & Transversal & $42,7 \%$ & $M, F$ & 1450 & $\begin{array}{l}\text { Inquérito } \\
\text { telefônico }\end{array}$ & $>18$ & $\begin{array}{l}\text { Sexo, idade, } \\
\text { escolaridade, status } \\
\text { civil, presença } \\
\text { de criança em } \\
\text { casa, importância } \\
\text { atribuída para a } \\
\text { facilidade do uso } \\
\text { de bicicleta no } \\
\text { deslocamento, } \\
\text { intenção para o uso } \\
\text { da bicicleta }\end{array}$ & $\begin{array}{l}\text { Disponibilidade de } \\
\text { bicicleta, uso de ciclovias, } \\
\text { proximidade dos destinos, } \\
\text { segurança na ciclovia e } \\
\text { posse de carro }\end{array}$ \\
\hline $\begin{array}{l}\text { Titze et al. } \\
(2010)^{9}\end{array}$ & Austrália & Transversal & $5,8 \%$ & $M, F$ & 1.795 & $\begin{array}{l}\text { Inquérito } \\
\text { domiciliar }\end{array}$ & $\begin{array}{c}40,0 \pm \\
11,9\end{array}$ & $\begin{array}{l}\text { Sexo, idade, } \\
\text { escolaridade } \\
\text { atitudes em relação } \\
\text { ao uso da bicicleta }\end{array}$ & $\begin{array}{l}\text { Posse de carro, acesso } \\
\text { a serviços, atratividade, } \\
\text { acessibilidade }\end{array}$ \\
\hline $\begin{array}{l}\text { Tin Tin et } \\
\text { al. }(2009)^{42}\end{array}$ & $\begin{array}{l}\text { Nova } \\
\text { Zelândia }\end{array}$ & Transversal & -- & $M, F$ & 2469 & $\begin{array}{l}\text { Inquérito } \\
\text { online }\end{array}$ & $>16$ & $\begin{array}{l}\text { Sexo, idade, } \\
\text { escolaridade }\end{array}$ & $\begin{array}{l}\text { Área de residência, } \\
\text { presença de ciclovia e } \\
\text { ciclofaixa, segurança } \\
\text { nos locais públicos, } \\
\text { redução da velocidade } \\
\text { dos veículos, estrutura } \\
\text { para ir de bicicleta para } \\
\text { o trabalho, cruzamentos } \\
\text { difíceis, custo do } \\
\text { combustível }\end{array}$ \\
\hline
\end{tabular}

\section{Fatores associados com o uso de bicicleta}

Foram identificados 57 fatores potencialmente associados com o uso de bicicleta (tabela 3). Entre os fatores individuais, sexo masculino $0^{4,7-10,18,20,29,39-41} \mathrm{e}$ prática de atividade física em níveis recomendados $(\geq 150 \mathrm{~min} / \mathrm{sem})^{2,12,28,29,35}$ apresentaram associação positiva conclusiva com o uso da bicicleta. Ainda, o trabalho de oito horas por dia ${ }^{10,12,29}$ apresentou associação negativa conclusiva com o desfecho.

Entre os fatores ambientais, acesso a ciclovias/ciclofaixas, ${ }^{929,40,42}$, densidade de ruass ${ }^{4,8,9}$, moradia urbana ${ }^{18,30,42}$ e disponibilidade de bicicleta no domicílio ${ }^{4,20,29,41}$ apresentaram associação positiva conclusiva com o uso de bicicleta. No entanto, distância para os destinos superiores a quatro $\mathrm{km}^{13,20,31,33}$, inclinação das ruas ${ }^{4,8,13}$ e posse de carro ${ }^{4,9,14,18,20,29,4}$ apresentaram associação negativa conclusiva com o desfecho. 
Por fim, idade, escolaridade, renda e segurança no tráfego apresentaram associações inconclusivas. Os demais 43 fatores apresentaram associações inconsistentes. Os estudos são apresentados de maneira detalhada na tabela 3.

TABELA 3 - Síntese das evidências sobre os fatores individuais e ambientais associados com o uso de bicicleta.

\begin{tabular}{|c|c|c|c|c|c|c|}
\hline & \multicolumn{3}{|c|}{ Associação } & \multirow{3}{*}{$\begin{array}{c}\% \text { de } \\
\text { estudos }\end{array}$} & \multirow{3}{*}{ Código* } & \multirow{3}{*}{ Associação } \\
\hline & \multicolumn{2}{|c|}{ Sim } & \multirow{2}{*}{ Não } & & & \\
\hline & Positiva & Negativa & & & & \\
\hline \multicolumn{7}{|c|}{ Fatores individuais (demográficos, psicológicos, cognitivos e emocionais) } \\
\hline Afastamento do trabalho por doença & & 43 & & $100 \%$ & ?? & Inconsistente \\
\hline Atende a recomendação de atividade física & $2,12,28,29,35$ & & 30 & $83 \%$ & + & Conclusiva \\
\hline Atitude em relação ao uso da bicicleta & 9,30 & & & $100 \%$ & ?? & Inconsistente \\
\hline Benefícios do uso da bicicleta & 11 & & & $100 \%$ & ?? & Inconsistente \\
\hline Comportamento ativo dos colegas & 14 & & & $100 \%$ & $? ?$ & Inconsistente \\
\hline Comportamento de ciclismo na comunidade & 4,33 & & & $100 \%$ & ?? & Inconsistente \\
\hline Escolaridade & $2,7,8,13,18,31,33$ & $4,32,42$ & $9,10,12,37$ & $50 \%$ & $?$ & Inconclusiva \\
\hline Estado civil solteiro & 2 & & 39 & $50 \%$ & $? ?$ & Inconsistente \\
\hline Falta de interesse & & 35 & & $100 \%$ & $? ?$ & Inconsistente \\
\hline Falta de tempo & & 35 & & $100 \%$ & $? ?$ & Inconsistente \\
\hline Força do hábito & 11,37 & & & $100 \%$ & $? ?$ & Inconsistente \\
\hline Idade & $9,14,33$ & $2,4,7,8,17,41,42$ & $12,30,32,37,39$ & $47 \%$ & $?$ & Inconclusiva \\
\hline IMC normal & 13 & & 37 & $50 \%$ & $? ?$ & Inconsistente \\
\hline Trabalho de oito horas & & $10,12,29$ & & $100 \%$ & - & Conclusiva \\
\hline Percepção de benefícios para a saúde & 11 & & & $100 \%$ & $? ?$ & Inconsistente \\
\hline Percepção de conforto & 33 & & & $100 \%$ & $? ?$ & Inconsistente \\
\hline Percepção de desconforto físico & & 13 & & $100 \%$ & $? ?$ & Inconsistente \\
\hline $\begin{array}{l}\text { Percepccão de modo impraticável de } \\
\text { transporte }\end{array}$ & & 14 & & $100 \%$ & $? ?$ & Inconsistente \\
\hline Percepção de pouco esforço fisiológico & 14 & & & $100 \%$ & $? ?$ & Inconsistente \\
\hline Percepção do benefício do modo ecológico & 35 & & & $100 \%$ & $? ?$ & Inconsistente \\
\hline Percepção positiva de saúde & 19 & & 33 & $50 \%$ & ?? & Inconsistente \\
\hline Percepção que o ciclismo é normal (cultural) & 33 & & & $100 \%$ & $? ?$ & Inconsistente \\
\hline Presença de morbidades & 19 & & & $100 \%$ & $? ?$ & Inconsistente \\
\hline Renda & 32 & $2,7,8,18$ & $4,10,12,37$ & $44 \%$ & $?$ & Inconclusiva \\
\hline Sexo masculino & $\begin{array}{c}4,7- \\
10,18,20,29,39-41\end{array}$ & & $12,13,37$ & $79 \%$ & + & Conclusiva \\
\hline Stress & 36 & & & $100 \%$ & $? ?$ & Inconsistente \\
\hline Exposição ao trânsito & 29 & & & $100 \%$ & $? ?$ & Inconsistente \\
\hline \multicolumn{7}{|l|}{ Fatores ambientais (físicos, sociais e natural) } \\
\hline Acessibilidade para ciclovia & $9,29,40,42$ & & 5,14 & $60 \%$ & + & Conclusiva \\
\hline Acesso a vestiário no local de trabalho & 34 & & 5 & $50 \%$ & $? ?$ & Inconsistente \\
\hline Apoio social & 13,35 & & & $100 \%$ & $? ?$ & Inconsistente \\
\hline Atratividade do bairro & 9 & & 14 & $50 \%$ & $? ?$ & Inconsistente \\
\hline Clima desfavorável & & 7,39 & & $100 \%$ & $? ?$ & Inconsistente \\
\hline Comprimento da ciclovia & 4 & & & $100 \%$ & $? ?$ & Inconsistente \\
\hline Conectividade de ciclovia & 14 & & & $100 \%$ & $? ?$ & Inconsistente \\
\hline Custo do combustível & 32,42 & & & $100 \%$ & ?? & Inconsistente \\
\hline
\end{tabular}




\begin{tabular}{|c|c|c|c|c|c|c|}
\hline & \multicolumn{3}{|c|}{ Associação } & \multirow{3}{*}{$\begin{array}{c}\% \text { de } \\
\text { estudos }\end{array}$} & \multirow{3}{*}{ Código* } & \multirow{3}{*}{ Associação } \\
\hline & \multicolumn{2}{|c|}{ Sim } & \multirow{2}{*}{ Não } & & & \\
\hline & Positiva & Negativa & & & & \\
\hline Densidade de ruas & $4,8,9$ & & & $100 \%$ & + & Conclusiva \\
\hline Densidade populacional & 8,31 & & & $100 \%$ & ?? & Inconsistente \\
\hline Disponibilidade de bicicleta & $4,20,29,41$ & & & $100 \%$ & + & Conclusiva \\
\hline Distância para o destino $>4 \mathrm{~km}$ & & $13,20,31,33$ & & $100 \%$ & - & Conclusiva \\
\hline Elevado Walkability & 17 & & & $100 \%$ & ?? & Inconsistente \\
\hline Expansão urbana & 37 & & 20 & $50 \%$ & ?? & Inconsistente \\
\hline Inclinação das ruas & & $4,8,13$ & 20 & $75 \%$ & - & Conclusiva \\
\hline Instalações para bicicleta no trabalho & 35 & & & $100 \%$ & ?? & Inconsistente \\
\hline Maior disponibilidade de destinos & 5,29 & & & $100 \%$ & $? ?$ & Inconsistente \\
\hline Mobilidade & 14 & & & $100 \%$ & ?? & Inconsistente \\
\hline Moradia urbana & $18,30,42$ & & 2 & $75 \%$ & + & Conclusiva \\
\hline Morar próximo do trabalho & 12,41 & & & $100 \%$ & $? ?$ & Inconsistente \\
\hline Noite & & 20 & & $100 \%$ & ?? & Inconsistente \\
\hline Poucos cruzamentos & 42 & & & $100 \%$ & $? ?$ & Inconsistente \\
\hline Posse de carro & & $4,9,14,18,20,29,41$ & & $100 \%$ & - & Conclusiva \\
\hline Presença de lojas & 29 & & & $100 \%$ & $? ?$ & Inconsistente \\
\hline Presença de parques & 31 & & & $100 \%$ & ?? & Inconsistente \\
\hline Redução da velocidade dos veículos & 8,9 & & 42 & $33 \%$ & $? ?$ & Inconsistente \\
\hline Satisfação com o bairro & & & 14 & $100 \%$ & ?? & Inconsistente \\
\hline Segurança do tráfego & 11,33 & 14 & 5,35 & $40 \%$ & $?$ & Inconclusiva \\
\hline Segurança em locais públicos & 42 & & & $100 \%$ & ?? & Inconsistente \\
\hline Segurança para estacionar a bicicleta & 14,34 & & & $100 \%$ & ?? & Inconsistente \\
\hline
\end{tabular}

*concordância das evidências: + associação positiva, com evidências em $\geqslant$ três estudos, concordância $\geqslant 60 \%$ = "conclusivos"; - Associação negativa com evidências em $\geqslant$ três estudos, concordância $\geqslant 60 \%=$ "conclusivos"; ? Concordância das evidências em $\geqslant$ três estudos, concordância < 60\% = "inconclusivos"; ?? Concordância das evidências < de três estudos = "Inconsistentes".

\section{DISCUSSÃO}

O objetivo principal desta revisão foi sintetizar as evidências sobre os fatores associados com o uso da bicicleta por adultos. Foram identificados 57 fatores potencialmente associados com este desfecho. Destes, três fatores individuais (sexo masculino, maior nível de atividade física e trabalho de oito horas) e sete fatores ambientais (acesso a ciclovias/ciclofaixa, densidade de ruas, distância até os destinos, inclinação das ruas, moradia urbana, posse de carros e disponibilidade de bicicleta no domicílio) apresentaram associação conclusiva com o uso de bicicletas.

Cerca de $80 \%$ dos estudos, que realizaram análises estratificadas por sexo,

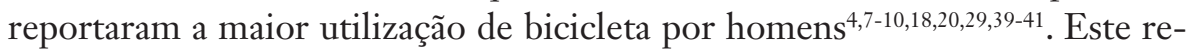
sultado pode ser, em parte, explicado por características relacionadas com as diferentes percepções entre homens e mulheres. Homens percebem maior segurança ${ }^{42}$ e um maior número de aspectos ambientais da vizinhança ${ }^{9,20}$. Enquanto as mulheres, além da diferente percepção de segurança, apresentam maior dificuldade em relação ao manejo e manutenção da bicicleta ${ }^{44}$. Estas informações podem auxiliar na compreensão da diferente relação entre sexo e uso da bicicleta, o que pode ser útil na elaboração de intervenções para 
aumentar o uso da bicicleta. Evidências apontam associação positiva entre percepção de segurança no trânsito, estética ${ }^{9}$ e presença de infraestrutura no bairro $^{29,40}$ com o uso de bicicleta, especialmente entre os grupos mais suscetíveis, como mulheres, crianças e idosos.

A associação conclusiva e positiva entre atividade física com o uso da bicicleta pode ser explicada por aspectos psicossociais ${ }^{2}$ e do estilo de vida ${ }^{12}$. Indivíduos fisicamente ativos apresentam percepção positiva de saúde ${ }^{19}$, maior apoio social $^{13,14}$, autoeficácia ${ }^{35}$, satisfação com a prática de atividades físicas em geral ${ }^{14,}$ assim como o conhecimento dos benefícios do deslocamento ativo ${ }^{11}$, o que poderia favorecer o uso de bicicleta. Assim, as intervenções poderiam ser direcionadas a indivíduos menos ativos e proporcionar, por exemplo, maior apoio social, satisfação com o uso de bicicleta e reforçar os seus benefícios para a saúde.

Trabalho de oito horas foi inversamente associado de forma conclusiva com o uso da bicicleta no deslocamento. Aspectos como a falta de tempo, a distância até o local de trabalho e a posse de carro, poderiam em parte, explicar este resultado. Evidências mostram que a implantação de estruturas no local de trabalho, como vestiário e estacionamento para bicicletas, podem favorecer o uso da bicicleta no deslocamento para o trabalho ${ }^{14,42}$.

Algumas características estruturais das cidades, como o acesso e conectividade de ciclovias/ciclofaixa ${ }^{9,29,14}$ e densidade de ruas $^{4}$, podem favorecer o uso da bicicleta. Outras, no entanto, como a inclinação das ruas ${ }^{4}$ e a maior distância até o destino ${ }^{20,33}$, dificultam este comportamento. Estas características, associadas com a posse e a utilização de $\operatorname{carros}^{4,9}$, podem diminuir o uso da bicicleta principalmente para o deslocamento para o trabalho. A indisponibilidade de bicicleta no domicílio pode contribuir para a menor intenção do seu uso como forma de deslocamento ou no lazer ${ }^{4,29,41}$. Uma alternativa de intervenção poderia ser a implantação de ciclovias/ciclofaixas bem conectadas, com rotas em regiões com maior densidade de ruas planas, o que poderia minimizar a percepção de distância dos usuários de bicicleta. Estes resultados poderiam ser potencializados pela redução dos impostos para a aquisição da bicicleta, facilitando a sua disponibilidade no domicílio.

Alguns fatores identificados na presente revisão não apresentaram associação conclusiva com o uso da bicicleta (exemplo: idade, escolaridade, renda e segurança no tráfego), sinalizando que este comportamento pode sofrer influências multifatoriais (aspectos intrapessoais, interpessoais e ambientais). Quarenta e três fatores apresentaram baixa consistência de associação sendo pouco estudados até o momento (menos de três estudos).

Algumas limitações devem ser consideradas para a adequada compreensão dos resultados desta revisão. Devido a diversos aspectos metodológicos dos estudos incluídos na revisão (medida do uso da bicicleta, variáveis de exposição, processo de amostragem, seleção dos participantes) não foi possível aplicar um instrumento para determinar a qualidade das evidências. Assim, a aplicação de um único instrumento poderia gerar escores imprecisos, o que ocasionaria o julgamento equivocado da qualidade das evidências. $\mathrm{O}$ critério de inclusão de estudos publicados somente em língua inglesa e portuguesa pode ter restringido evidências publicadas em outro idioma. Por fim, a maioria das evidências foi baseada em estudos transversais, em que não é possível identificar a relação causal entre as variáveis. 


\section{CONCLUSÃO}

Os fatores individuais sexo masculino, nível de atividade física, trabalho de oito horas; e os fatores ambientais acesso a ciclovias/ciclofaixas, maior densidade de ruas, moradia urbana, posse de carro, disponibilidade de bicicleta, menor distância até o destino e a inclinação das ruas apresentaram associação conclusiva com o uso de bicicleta.

Estes resultados podem auxiliar na elaboração de políticas públicas que favoreçam o uso de bicicleta pela população. Por exemplo, as mulheres podem ser incentivadas a usar a bicicleta com melhoria na segurança pública e leis rígidas de proteção ao ciclista, enquanto os trabalhadores podem se beneficiar com a implementação de estruturas no local de trabalho que facilitem o uso da bicicleta. A comunidade em geral pode ser beneficiada pela implementação de ciclovia/ciclofaixas, com melhor conexão por meio de rotas planejadas com menor inclinação e que reduzam a percepção de distância. A redução de impostos como subsídios para aquisição de bicicletas pode servir como incentivo à redução do uso do carro como principal meio de deslocamento.

Futuros estudos devem analisar o impacto que as políticas públicas de promoção ou as modificações do ambiente das cidades podem exercer sobre o uso da bicicleta em diferentes faixas etárias.

\section{Financiamento}

M Kienteka e RC Fermino receberam bolsa da CAPES (Coordenação de Aperfeiçoamento de Pessoal de Nível Superior) durante a condução da revisão na Pós-Graduação.

\section{Contribuição dos autores}

M Kienteka foi responsável pela concepção do estudo, busca das referências, leitura, avaliação e extração das informações dos estudos e liderou a redação do manuscrito em todas as suas etapas. RC Fermino participou na busca preliminar das referências e na extração das suas informações, além de ter colaborado na redação e revisão crítica do manuscrito em todas as suas etapas. RS Reis foi responsável pela concepção do estudo e revisão crítica do manuscrito.

\section{REFERÊNCIAS}

1. Wen LM, Rissel C. Inverse associations between cycling to work, public transport, and overweight and obesity: findings from a population based study in Australia. Prev Med 2008;46(1):29-32.

2. Butler GP, Orpana HM, Wiens AJ. By your own two feet: factors associated with active transportation in Canada. Can J Public Health 2007;98(4):259-264.

3. Woodcock J, Banister D, Edwards P, Prentice AM, Roberts I. Energy and transport. Lancet 2007; 370 (9592): 1078-1088.

4. Cervero R, Sarmiento O, Jacoby E, Gomez LF, Neiman A. Influences of built environments on walking and cycling: lessons from Bogotá. Int J Sust Transp 2009; 3 (4): 203-226.

5. Parra DC, Hoehner CM, Hallal PC, et al. Perceived environmental correlates of physical activity for leisure and transportation in Curitiba, Brazil. Prev Med 2011.

6. Reis RS, Hino AAF, Parra DC, Hallal PRC, Brownson RC. Bicycling and walking for transportation in three Brazilian cities. Am J Prev Med 2013; 44 (2): e9-e17.

7. Winters M, Friesen MC, Koehoorn M, Teschke K. Utilitarian Bicycling: a multilevel analysis of climate and personal influences. Am J Prev Med 2007; 32 (1): 52-58. 
8. Winters M, Brauer M, Setton EM, Teschke K. Built environment influences on healthy transportation choices: bicycling versus driving. J Urban Health 2010; 87 (6): 969-993.

9. Titze S, Giles-Corti B, Knuiman MW, et al. Associations between intrapersonal and neighborhood environmental characteristics and cycling for transport and recreation in adults: baseline results from the RESIDE study. J Phys Act Health 2010; 7(4): 423-431.

10. Owen N, De Bourdeaudhuij I, Sugiyama T, et al. Bicycle use for transport in an Australian and a Belgian city: associations with built-environment attributes. J Urban Health 2010;87(2):189-198.

11. Heinen E, Maat K, Wee Bv. The role of attitudes toward characteristics of bicycle commuting on the choice to cycle to work over various distances. Transp Res Part D: Transport and Environment 2010;16(2):102-109.

12. Engbers LH, Hendriksen IJ. Characteristics of a population of commuter cyclists in the Netherlands: perceived barriers and facilitators in the personal, social and physical environment. Int J Behav Nutr Phys Act 2010;7:89.

13. Titze S, Stronegger WJ, Janschitz S, Oja P. Environmental, social, and personal correlates of cycling for transportation in a student population. J Phys Act Health 2007;4(1):66-79.

14. Titze S, Stronegger WJ, Janschitz S, Oja P. Association of built-environment, socialenvironment and personal factors with bicycling as a mode of transportation among Austrian city dwellers. Prev Med 2008;47(3):252-259.

15. Sener IN, Eluru N, Bhat CR. An analysis of bicycle route choice preferences in Texas, US. Transportation 2009;36(5):511-539.

16. Pikora T, Giles-Corti B, Bull F, Jamrozik K, Donovan R. Developing a framework for assessment of the environmental determinants of walking and cycling. Soc Sci Med 2003;56(8):1693-1703.

17. Owen N, Cerin E, Leslie E, et al. Neighborhood walkability and the walking behavior of Australian adults. Am J Prev Med 2007;33(5):387-395.

18. Plaut PO. Non-motorized commuting in the US. Transp Res Part D: Transport and Environment 2005;10(5):347-356.

19. Huy C, Becker S, Gomolinsky U, Klein T, Thiel A. Health, medical risk factors, and bicycle use in everyday life in the over-50 population. J Aging Phys Act 2008;16(4):454-464.

20. Cervero R, Duncan M. Walking, bicycling, and urban landscapes: evidence from the San Francisco Bay Area. Am J Public Health 2003;93(9):1478-1483.

21. Sallis JF, Floyd MF, Rodriguez DA, Saelens BE. Role of built environments in physical activity, obesity, and cardiovascular disease. Circulation 2012;125(5):729-737.

22. Sampaio RF, Mancini MC. Estudos de revisão sistemática: um guia para síntese criteriosa da evidência científica; Systematic review studies: a guide for careful synthesis of thescientific evidence. Rev. bras. Fisioter 2007;11(1):83-89.

23. Pucher J, Dill J, Handy S. Infrastructure, programs, and policies to increase bicycling: An international review. Prev Med 2010;50:S106-S125.

24. Sallis JF, Linton L, Kraft MK. The first Active Living Research Conference: growth of a transdisciplinary field. Am J Prev Med 2005;28(2 Suppl 2):93-95.

25. Pucci GC, Rech CR, Fermino RC, Reis RS. Associação entre atividade física e qualidade de vida em adultos. Rev SaúdePública 2012;46(1):166-179.

26. Sallis JF, Prochaska JJ, Taylor WC. A review of correlates of physical activity of children and adolescents.Med Sci Sports Exerc. 2000;32(5):963-75.2000.

27. Fermino RC, Reis RS. Variáveis individuais, ambientais e sociais associadas com o uso de espaços públicos abertos para a prática de atividade física: uma revisão sistemática. Rev Bras Ativ Fís Saúde 2013; 18(5):523-535.

28. Sharpe PA, Granner ML, Hutto B, Ainsworth BE. Association of environmental factors to meeting physical activity recommendations in two South Carolina counties. Am J Health Promot 2004;18(3):251-257.

29. Moudon AV, Lee C, Cheadle AD, et al. Cycling and the built environment, a US perspective. Transp Res Part D: Transport and Environment 2005;10(3):245-261.

30. Heesch KC, Han JL. Associations between demographic, perceptual, and behavioral factors and support for policies encouraging active transport. J Phys Act Health 2007;4(3):261-277. 
31. Zahran S, Brody SD, Maghelal P, Prelog A, Lacy M. Cycling and walking: Explaining the spatial distribution of healthy modes of transportation in the United States. Transp Res Part D: Transport and Environment 2008;13(7):462-470.

32. Rashad I. Associations of cycling with urban sprawl and the gasoline price. Am J Health Promot 2009;24(1):27-36.

33. Xing Y, Handy SL, Mokhtarian PL. Factors associated with proportions and miles of bicycling for transportation and recreation in six small US cities. Transp Res Part D: Transport and Environment 2010;15(2):73-81.

34. Kaczynski AT, Bopp MJ, Wittman P. Association of workplace supports with active commuting. Prev Chronic Dis 2010;7(6):A127.

35. De Geus B, De Bourdeaudhuij I, Jannes C, Meeusen R. Psychosocial and environmental factors associated with cycling for transport among a working population. Health Educ Res 2008;23(4):697.

36. Asztalos M, Wijndaele K, De Bourdeaudhuij I, et al. Specific associations between types of physical activity and components of mental health. J Sci Med Sport 2009;12(4):468-474.

37. Bruijn GJ, Kremers SP, Singh A, Van Den Putte B, Van Mechelen W. Adult active transportation: adding habit strength to the theory of planned behavior. Am J Prev Med 2009;36(3):189-194.

38. Hendriksen IJ, Simons M, Garre FG, Hildebrandt VH. The association between commuter cycling and sickness absence. Prev Med 2010;51(2):132-135.

39. Merom D, Bauman A, Vita P, Close G. An environmental intervention to promote walking and cycling--the impact of a newly constructed Rail Trail in Western Sydney. Prev Med 2003;36(2):235-242.

40. Garrard J, Rose G, Lo SK. Promoting transportation cycling for women: the role of bicycle infrastructure. Prev Med 2008;46(1):55-59.

41. Rissel C, Merom D, Bauman A, et al. Current cycling, bicycle path use, and willingness to cycle more-findings from a community survey of cycling in southwest Sydney, Australia. J Phys Act Health 2010;7(2):267-272.

42. Tin Tin S, Woodward A, Thornley S, et al. Cyclists' attitudes toward policies encouraging bicycle travel: findings from the Taupo Bicycle Study in New Zealand. Health Promot Int 2009;25(1):54-62.

43. Hendriksen IJM, Zuiderveld BOB, Kemper HANCG, Bezemer P. Effect of commuter cycling on physical performance of male and female employees. Med Sci Sports Exerc 2000;32(2):504.

44. Heesch KC, Sahlqvist S, Garrard J. Gender differences in recreational and transport cycling: a cross-sectional mixed-methods comparison of cycling patterns, motivators, and constraints. Int J Behav Nutr Phys Act, 2012. 9(1):106.

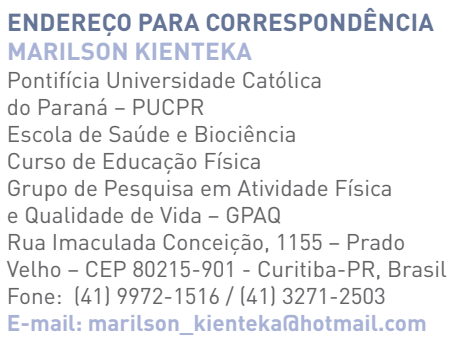

